\title{
A modified drought index for WMO RA VI
}

\author{
S. Pietzsch and P. Bissolli \\ Deutscher Wetterdienst, Offenbach, Germany
}

Received: 4 January 2011 - Revised: 12 August 2011 - Accepted: 30 August 2011 - Published: 20 October 2011

\begin{abstract}
Drought is a phenomenon which can cause large economical impact even in Europe. To assess the magnitude and the spatial extension of drought events, it is important to have a standardized drought index which is applicable for a large climatically heterogeneous region like Europe or the WMO RA VI Region (Europe and the Middle East). Such an index should describe the drought phenomenon adequately, but it should also be derivable from meteorological quantities which are easily and timely available in whole Europe.

In a first investigation, some candidates for drought indices were chosen, compared and assessed for applicability in whole Europe. The most appropriate one seems to be the widely known Standardized Precipitation Index (SPI) which is a standardized and handy measurement of drought for any location and requires nothing but precipitation data. However, it has turned out that for some places in the RA VI Region, notably in arid regions in summer, the SPI does not always provide reasonable or easily interpretable results.

For that reason, some modifications of the SPI have been tried out and tested statistically. It seems that the gamma distribution of precipitation which is used for computation of the SPI is in fact the most appropriate one and other distributions have not improved the results substantially. On the other hand a so called zero correction, which sets very small precipitation totals to dry values, only dependent on the precipitation distribution, but independent on the individual location delivers more reasonable results.

Maps of the new modified drought index and its anomalies from the climate normal are produced quasioperationally and distributed via the Internet each month. The drought monitoring is part of the monitoring programme of the WMO RA VI Pilot Regional Climate Centre on Climate Monitoring (RCC-CM) hosted by the German Meteorological Service (Deutscher Wetterdienst, DWD), and the maps can be found on its present RCC-CM platform (http://www.dwd.de/rcc-cm).
\end{abstract}

\section{Introduction}

Drought is a phenomenon which can appear in many parts of the globe (Wilhite, 2000) and can cause large economic impacts even in Europe. The spatio-temporal evolution and characteristics of large-scale European droughts have been investigated recently, e.g. by Parry et al. (2010) and Hannaford et al. (2011), including linking droughts to weather types (Fleig et al., 2011) and analysing drought trends for the Mediterranean e.g. by Sousa et al. (2011); further numerous studies have been carried out on a national or subregional scale.

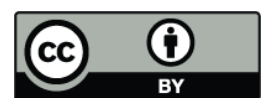

Correspondence to: P. Bissolli (peter.bissolli@dwd.de)
To assess the magnitude and the spatial extent of drought events, it is important to have a standardized drought index which is applicable for a large climatically heterogeneous region like Europe or the WMO RA VI Region (Europe and the Middle East). Such an index should describe the drought phenomenon adequately, but it should also be derivable from meteorological quantities which are easily and timely available for the whole of Europe. It is desirable to have one simple index to show the drought characteristics of the wide and disparate region. The aim of the investigation is to produce an operational system to monitor drought conditions, showing their variability in time and space. It should be an index which is easy to calculate and apply to the whole region so that individual sub-regions can be easily compared to each other. This is possible for standardized indices which are generally applicable for each location within the Region 
and do not contain any local or sub-regional specifics or, in case there are any, they need to be adapted or modified. There were many previous approaches for deriving and using drought indices, e.g. by Parry et al. (2010). Here, the approach is to create a drought index by choosing an already existing and widely used index which is applicable for most of the WMO RA VI Region, and by introducing modifications for those parts of the Region where improvement of the index is needed due to their special climatic characteristics. Such an index can provide an overview of drought conditions and its variability in space and time operationally.

As agricultural and hydrological droughts need relatively long timescales to be indexed, McKee et al. (1993) originally calculated a drought index, the SPI (see Sect. 2), for 3-, 6-, 12-, 24- and 48-month timescales. This paper concentrates on a monthly timescale, which can be used to characterise meteorological drought. Another reason for having chosen that time scale is that the aim of this paper is the construction of a drought index for monthly climate monitoring, to enable a comparison with other monthly climate monitoring products such as temperature and precipitation anomalies.

\section{Comparison of drought indices}

In a first investigation (Pietzsch, 2009), three drought indices were chosen, compared and assessed for applicability to the whole of Europe. These were the Aridity Index according to de Martonne (1950) (Precipitation/[Temperature + 10]), the Climatic Water Balance (Müller-Westermeier, 2005) (Precipitation Evaporation according to Wendling, 1995) and the Standardized Precipitation Index (McKee et al., 1993) (precipitation anomalies shown as multiples of the standard deviation of an adapted theoretical distribution; here the gamma distribution and the reference period 1971-2007). The indices were calculated for every month of the years 2002 to 2006 based on various data sources (see Pietzsch, 2009 for more details) and mapped using a GIS (Geographical Information System) on a regular grid with a resolution of $0.5 \times 0.5$ degrees latitude/longitude. The results were that the Aridity Index according to de Martonne does not give any useful results for temperatures near or below -10 degrees Celsius and so cannot be applied to the whole of WMO RA VI. The Climatic Water Balance does not show extremes in an adequate way, because it expresses drought events too weakly. The most appropriate drought index seems to be the widely known Standardized Precipitation Index (SPI) which is a standardized and handy measurement of drought for any location and only requires precipitation data. However, for some places in the RA VI Region, notably in arid regions in summer such as southern Spain and the Middle East, the SPI does not always provide reasonable or easily interpretable results. It is problematic to calculate the SPI for very arid regions because precipitation near zero can cause misleading high or low SPI values (Lloyd-Hughes and Saunders,
2002). These problems appear especially for the time scale of 1 month when trying to describe regions in which aridity is normal during certain months (NDMC, 2011). In the case of zero precipitation during the whole period considered it is not possible to calculate any SPI value. And in regions with very little precipitation, the SPI misleadingly shows very high (humid) values.

\section{SPI modifications}

For that reason, some modifications of the SPI have been tried out and tested statistically using the gridded precipitation data of the Global Precipitation Climatology Centre (GPCC, full data reanalysis product version 4, spatial resolution $0.5 \times 0.5^{\circ}$; Schneider et al., 2008). First a choice of theoretical distributions (the binomial distribution, the exponential distribution, the gamma distribution, the Poisson distribution and the Weibull distribution) was applied to precipitation data from the problematic arid regions. Therefore 51 grid points in Portugal, southern Italy, Greece, Cyprus, Turkey, Lebanon, Syria, Israel and Jordan were chosen and the corresponding empirical precipitation distributions of August in years 1971 to 2007 were adapted to each of the theoretical distributions. Then the theoretical distributions were compared to the real precipitation distributions using the Chisquare test. It appeared reasonable to test a variety of distributions to find out whether there is a distribution function beside the widely accepted gamma distribution (McKee et al., 1993) that better fits the precipitation distribution in the arid regions. In that case the distribution function of the SPI could have been modified. In regions with values of only 0 or $1 \mathrm{~mm}$ precipitation the binomial distribution fitted very well. In the wetter regions the gamma distribution provided the best results but also the exponential distribution showed quite good results. All in all, however, the gamma distribution was the most suitable one with the lowest Chi-square values, which means the highest similarity to the real precipitation distributions. In a second step the distributions of the calculated SPI-values for the grid points mentioned above were compared with the normal distribution via the Chi-square test. This examination showed that none of these SPI distributions was normally distributed. Based on these findings, various correction approaches for the SPI were created and tested. One version was changing the distribution function and recalculating the SPI. Here a combination of the exponential distribution and the gamma distribution was used. Another model was a weighted addition of another distribution function to the existing SPI. Furthermore a socalled zero correction, in terms of setting very small precipitation totals to dry SPI values, was used to solve the problem that the present SPI does not adopt dry values in cases of no precipitation and an inappropriate distribution as in arid regions. Every variation of modification, with and without zero correction, was validated via comparison of Chi-square 

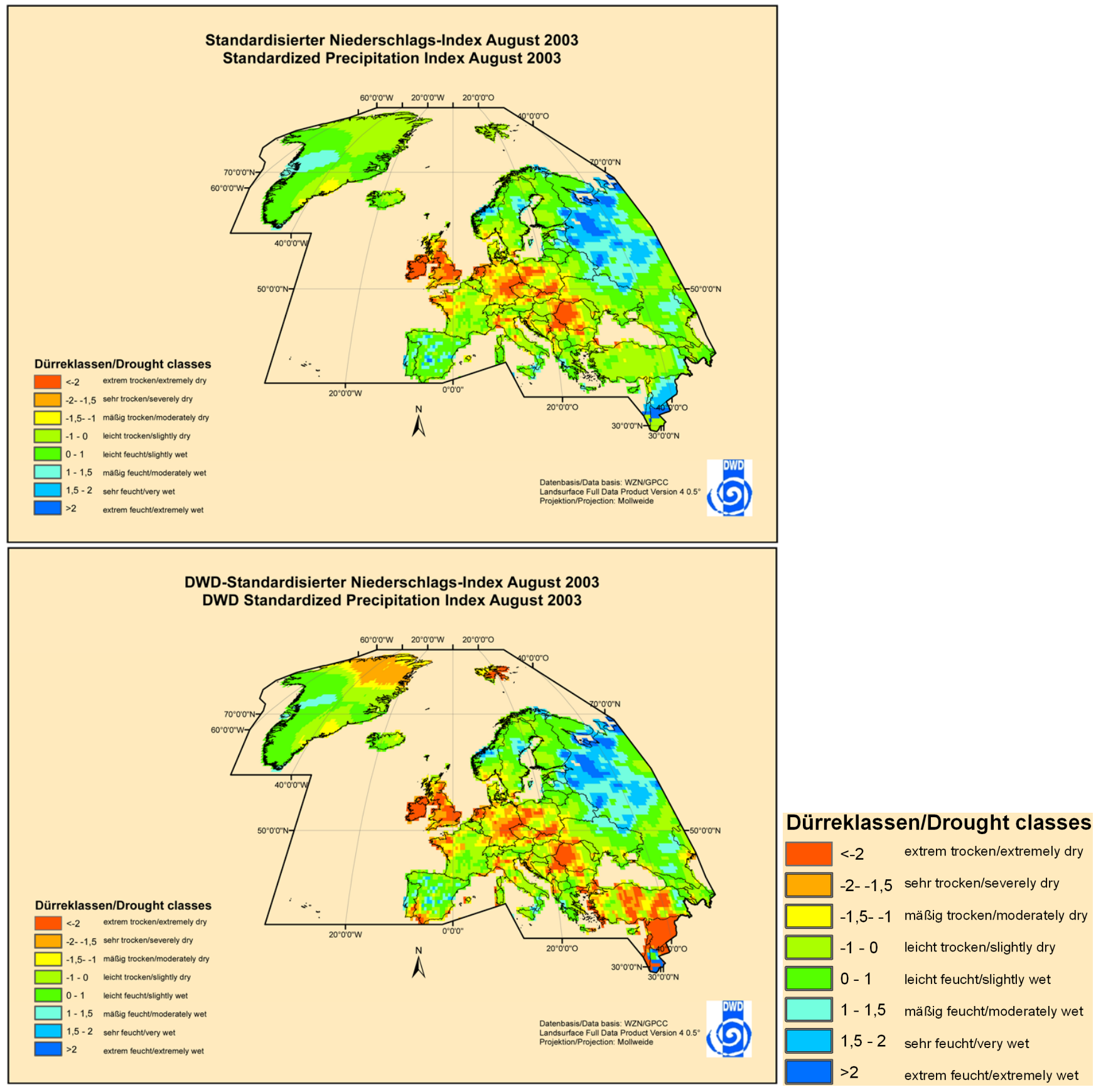

Figure 1. Comparison of the SPI (upper map) and the SPI DWD (lower map).

values. Here again, the Chi-square test was applied to find out whether the different correction approaches were normally distributed (by comparing the drought index distributions with a theoretical normal distribution); or rather which of them was the most normally distributed one. It seems that the gamma distribution of precipitation which is used for computation of the SPI is in fact the most appropriate one and other distributions have not improved the results substantially. On the other hand, the zero correction, only dependent on the precipitation distribution, but not explicitly dependent on the individual location delivers more reasonable results by making sure that zero precipitation yields low SPI-values for each location. To have a comparison and to get the longterm mean monthly parameters of the gamma distribution $(\alpha$, $\beta$, and $p 0=$ the relative frequency for zero precipitation) for further calculations, first the original SPI was computed for the years 1901-2009 for every grid point of WMO RA VI using the function: 
Table 1. Comparison of the SPI and the SPI DWD for several grid points.

\begin{tabular}{|c|c|c|c|c|}
\hline \multirow[t]{2}{*}{ Grid Point } & \multicolumn{2}{|c|}{ Precipitation August (mm) } & \multicolumn{2}{|c|}{ Drought Index August 2003} \\
\hline & $1971-2007$ & 2003 & SPI & SPI DWD \\
\hline $02.5^{\circ} \mathrm{W} 39.5^{\circ} \mathrm{N}$ (Spain) & 14.7 & 31.0 & 1.2 & 1.2 \\
\hline $07.5^{\circ} \mathrm{W} 40.5^{\circ} \mathrm{N}$ (Spain) & 12.9 & 30.0 & 1.2 & 1.0 \\
\hline $21.0^{\circ}$ E $38.0^{\circ} \mathrm{N}$ (Greece) & 10.4 & 19.0 & 0.9 & 0.7 \\
\hline $35.5^{\circ}$ E $33.5^{\circ} \mathrm{N}$ (Middle East) & 0.3 & 0.0 & 1.2 & -1.9 \\
\hline
\end{tabular}

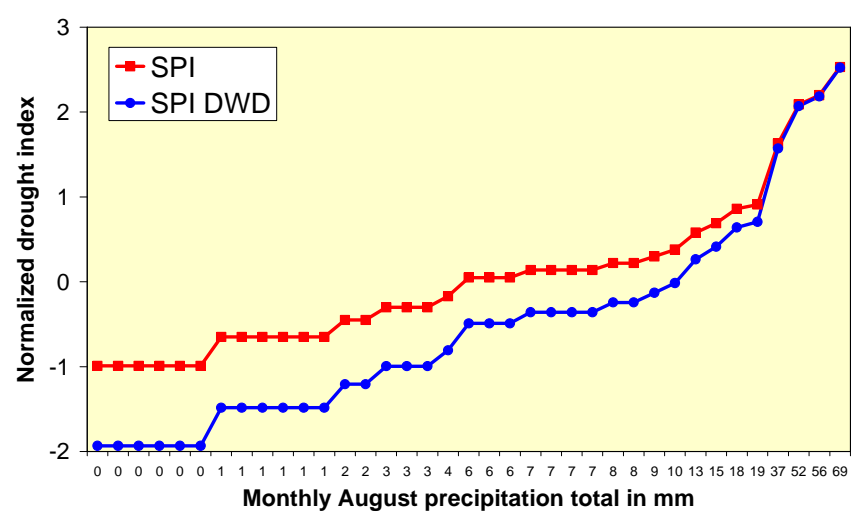

Figure 2. SPI and SPI DWD as function of monthly precipitation in August 1971-2007 at a grid point over Greece $\left(21.0^{\circ}\right.$ E $\left.38.0^{\circ} \mathrm{N}\right)$. Precipitation totals for each year of that period are displayed in ascending order. Parameters in this example: number of years $n=37$, relative frequency of zero precipitation $p 0=6 / n=0.162$.

$F(x)=(1-p 0) \cdot \Gamma(x, \alpha, \beta)$ for $x>0$

$F(x)=p 0$ for $x=0$

$x=$ precipitation total over the chosen time interval (e.g. 1 month); $p 0=$ relative frequency for zero precipitation total $(x=0)=$ number of years with zero precipitation in the given time span divided by the total number of evaluated years.

The cumulative probability, $F(x)$, is then transformed to the standard normal random variable $Z$ with mean zero and variance of one, which is the value of the SPI (http://ccc. atmos.colostate.edu/pub/spi.pdf).

Afterwards the most reasonable correction approaches for the SPI were calculated. By successive trying, correcting and modifying the various solutions, the final solution, we call it SPI DWD, was developed:

$$
\begin{aligned}
& \text { SPI DWD }=\text { SPI }+(\operatorname{NORMINV~}(1 / n) \operatorname{NORMINV}(p 0)) . \\
&(1-p(\mathrm{SPI})) /(1-p 0)
\end{aligned}
$$

$n=$ Number of evaluated years; NORMINV( $)=$ Inverse function of the standardized normal distribution; $p 0=$ Relative frequency for zero precipitation as in the original SPI $p(\mathrm{SPI})=$ Value of the distribution function of the standardized normal distribution for $z=\mathrm{SPI}$; for $x=0 \mathrm{~mm}$ : NORMINV $(1 / n)$ (e.g. for $n=107=-2.3=$ extremely dry);

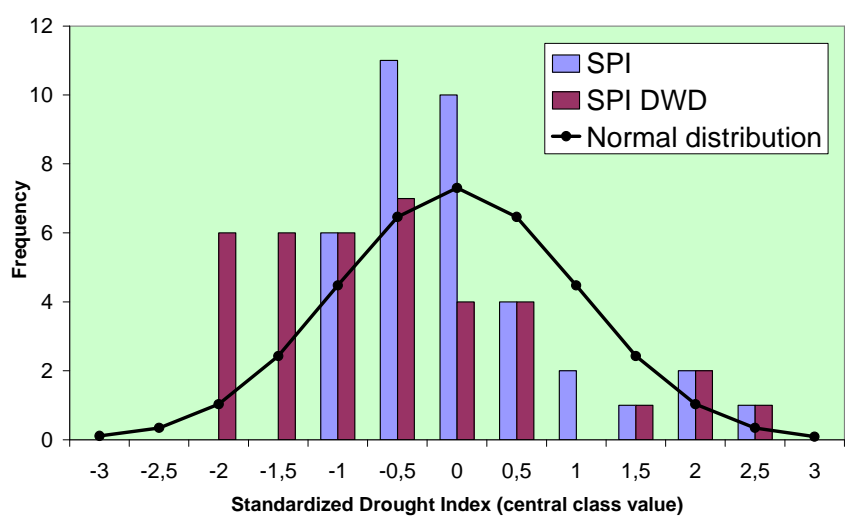

Figure 3. Frequency distribution of the SPI and the SPI DWD for the same data as in Fig. 2, compared with the corresponding normal distribution.

for $x>0 \mathrm{~mm}$ : the higher the rainfall, the greater $p(\mathrm{SPI})$, the smaller the difference SPI DWD - SPI, and the smaller the correction; for $p 0=0$ : SPI DWD $=\mathrm{SPI}$.

\section{Results}

The advantages of the new SPI DWD are that the arid regions in summer now can be correctly represented (see Fig. 1), that there is a smooth value transition from zero precipitation to little precipitation and that there are hardly any differences to the original SPI in case of higher precipitation.

The correction is only high for the extremely dry regions. In areas where no zero precipitation values appear, the SPI DWD is equal to the SPI and no correction is made. The smaller the precipitation is in a region, the higher is the difference between the SPI DWD and the SPI, so that now the regions with very little precipitation correctly show low (dry) SPI-values. In case of zero precipitation NORMINV $(1 / n)$ is set (e.g. for $107 \mathrm{yr}(n=107)$ a SPI value of -2.3 is the outcome). The complexity of the correction results from the aim of achieving the smooth value transition from zero precipitation to little precipitation.

The table above (Table 1) shows a comparison of the SPI and the SPI DWD for several grid points. It becomes evident that the difference between the two indices is quite small for the grid points in Spain and Greece, which do not have an 
extremely small long-term average (here August 1971-2007) precipitation total. The correction via the SPI DWD becomes clear, looking at the very dry grid point in the Middle East. Here the long-term precipitation average is $0.3 \mathrm{~mm}$ and the August 2003 value, with $0 \mathrm{~mm}$ precipitation, is even smaller. Therefore, a dry SPI value should be expected, but the original SPI index shows the "moderately wet" value of 1.2. The SPI DWD calculation, on the contrary, yields a more reasonable value of -1.9 , which means "severely dry".

The graph in Fig. 2 shows how the SPI and the SPI DWD behave over the years 1971 to 2007 at one of the 51 tested grid points mentioned in Sect. 3 with 6 of the $37 \mathrm{yr}$ having zero precipitation in August. In contrast to SPI, SPI DWD shows larger negative values close to -2 (severely/extremely dry) for zero precipitation and small totals, whereas for high precipitation the difference is close to zero since the original SPI already yields high values $>2$ which means extremely wet.

The disadvantage of the SPI DWD is that the values of its distribution are still not normally distributed in arid regions (see Fig. 3). However, in this case even the original SPI is not normally distributed, because the distribution of the precipitation values is inappropriate, since there are numerous small precipitation values. To achieve the aim of correctly representing very small precipitation values via very small SPI values we had to drop the normal distribution.

\section{Production of time series and operational maps}

The SPI DWD was computed for every month of the years 1901-2010 and the whole WMO RA VI. Since the GPCC full data reanalysis product presently was only available until 2007, the GPCC monitoring product had been used instead for the years since 2008 . The monitoring product has a lower data density and a coarser resolution $\left(1 \times 1^{\circ}\right)$ compared to the full data product, but the gridded data are available two months after the actual month. For near real time monitoring, another GPCC product (first guess product) is available around 5 days after completion of the month, but with even lower data density and lower quality control level compared to the GPCC monitoring product (Schneider et al., 2008).

The index was mapped using a GIS from 2005 onwards. Maps of the new modified drought index and its anomalies from the climate normal are produced quasi-operationally and distributed via the Internet each month. The drought monitoring is part of the monitoring programme of the WMO RA VI Pilot Regional Climate Centre on Climate Monitoring (RCC-CM) hosted by the German Meteorological Service (Deutscher Wetterdienst, DWD), and the maps can be found on its present platform http://www.dwd.de/rcc-cm.

Acknowledgements. Sincere thanks are given to the GPCC for providing the gridded precipitation data. The guest editor and three anonymous reviewers contributed some very constructive comments on the paper.
Edited by: J. Prior

Reviewed by: three anonymous referees

\section{SC nat $\$$ The publication of this article is sponsored by the Swiss Academy of Sciences.}

\section{References}

de Martonne, E.: Traite de géographie physique. Notions générales, climat, hydrogeographie, 8th edn., Colin, Paris, 1950 (in French).

Fleig, A. K., Tallaksen, L. M., Hisdal, H., and Hannah, D. M.: Regional hydrological drought in north-western Europe: linking a new Regional Drought Area Index with weather types, Hydrol. Process., 25, 1163-1179, doi:10.1002/hyp.7644, 2011.

Hannaford, J., Lloyd-Hughes, B., Keef, C., Parry, S., and Prudhomme, C.: Examining the large scale spatial coherence of European drought using regional indicators of precipitation and streamflow deficit, Hydrol. Process., 25, 1146-1162, doi:10.1002/hyp.7725, 2011.

Lloyd-Hughes, B. and Saunders, M. A.: A drought climatology for Europe, Int. J. Climatol., 22, 1571-1592, 2002.

McKee, T. B., Doesken, N. J., and Kleist, J.: The relationship of drought frequency and duration to time scales, Proceedings of the Eighth Conference on Applied Climatology, Anaheim, California, 1993.

Müller-Westermeier, G.: Die Klimatische Wasserbilanz, in: Deutscher Wetterdienst (2005): Klimaatlas Bundesrepublik Deutschland 4, Offenbach am Main, 2005 (in German).

National Drought Mitigation Center (NDMC): Interpretation of 1-Month Standardized Precipitation Index Map, http://drought.unl.edu/MonitoringTools/ClimateDivisionSPI/ Interpretation/1month.aspx, 2011.

Parry, S., Prudhomme, C., Hannaford, J., and Lloyd-Hughes, B.: Examining the spatio-temporal evolution and characteristics of large-scale European droughts, in: Role of Hydrology in Managing Consequences of a Changing Global Environment, Proceedings of the BHS Third International Symposium, edited by: Kirby, C., Newcastle, British Hydrological Society, 135-142, 2010.

Pietzsch, S.: Raum-zeitliche Analyse von Dürreperioden in der WMO Region VI (Europa und Naher Osten), Diploma thesis, Johannes Gutenberg University, Mainz, 2009 (in German).

Schneider, U., Fuchs, T., Meyer-Christoffer, A., and Rudolf, B.: Global Precipitation Climatology Centre (GPCC): Global Precipitation Analysis Products of the GPCC, Deutscher Wetterdienst Offenbach am Main, internet publication: http://gpcc.dwd. de, 2008.

Sousa, P. M., Trigo, R. M., Aizpurua, P., Nieto, R., Gimeno, L., and Garcia-Herrera, R.: Trends and extremes of drought indices throughout the 20th century in the Mediterranean, Nat. Hazards Earth Syst. Sci., 11, 33-51, doi:10.5194/nhess-11-33-2011, 2011.

Wendling, U.: Berechnung der Gras-Referenz-Verdunstung mit der FAO Penman-Monteith-Beziehung, Wasserwirtschaft 85E, H.12, 602-660, 1995 (in German).

Wilhite, D. A.: Drought a global assessment, Vol. I, London, 2000. 